\section{Research is the seed of future prosperity}

As scientists, journalists and policy-makers gather in Dublin this week for the Euroscience Open Forum (www.esof.eu), we need to answer a question that I have often been asked during my five years as chief scientific adviser to the Irish government. Why should a small country spend scarce taxpayers' money on research and development (R\&D) - particularly during a recession, when every extra euro is borrowed from the next generation?

The question contains its own answer: today's $\mathrm{R} \& \mathrm{D}$ is an investment in the wealth and welfare of future generations. This is why the Irish government treats public spending on $R \& D$ as capital expenditure, as it does for roads and schools.

The case for public $R \& D$ is laid out in a 2006 World Bank report, Where is the Wealth of Nations? Most of the wealth in all countries is in the intellectual capital of individuals, firms and institutions. In developed countries, including Ireland, intellectual capital generally exceeds $80 \%$ of total wealth.

Many nations are recalibrating their R\&D investment to help pull themselves out of the economic downturn. Spain, for example, is cutting funds. Finland and Ireland are among those boosting or protecting their science budgets in the interest of long-term growth.

A broad-based steering group has identified 14 priority areas for future R\&D investment in Ireland. Most relate to strong industrial sectors (information technology, pharmaceuticals, health, high-tech services) and to resources (energy, food, agricultural, marine). This economic focus is compatible with strong support for basic science in the priority areas, which is what industrial partners (who contribute two-thirds of the nation's $R \& D$ spending) say that they want.
Two further important sectors should, in my view, command perhaps one-third of public $\mathrm{R} \& \mathrm{D}$ investment in general. One is research to underpin policy, such as applied studies in environment, health, social science and economics. The other is research not directly relevant to economic or policy goals, such as astronomy, particle physics or the humanities, that can enrich our culture and national reputation. Patrick Cunningham Dublin, Ireland.patrick.cunningham@ chiefscientificadviser.ie

\section{A bleak day for the environment}

It was a dark day for environmental science and policy in Canada on 29 June.

The country's Conservative Party has been steadily dismantling environmental protection since winning a majority government last year (see, for example, Nature http://doi.org/h2v; 2012). Further alarming changes to environmental laws were concealed in a 'budget bill' that was ratified by the Senate on 29 June.

For example, the Canadian Environmental Assessment Act has been replaced by a weaker law that reduces government oversight of the environmental impact of a proposed pipeline from the Alberta oil sands to tankers off British Columbia. Canada's Fisheries Act now allows for more pollution and no longer protects fish habitats, except for fisheries. The National Round Table on the Environment and the Economy, which provides independent scientific advice on sustainable development, will be dissolved in March 2013. A finance committee that had no scientific or public input has decided that this massive legislative overhaul could proceed as written.

Globally significant research facilities have already been axed, including the renowned
Experimental Lakes Area and the Polar Environment Atmospheric Research Laboratory in the high Arctic. Scientific agencies such as Fisheries and Oceans Canada (DFO), Environment Canada and Parks Canada have had to sack most of the personnel responsible for habitat management and monitoring, including those in the DFO's marine-pollution programme.

The new legislative framework marginalizes science in environmental management, and could do irreparable harm to the environment and the economy it supports. Such tactics match Canada's intransigence on climate change: the same bill made it the first country to pull out of the Kyoto agreement.

John D. Reynolds, Isabelle M. Côté, Brett Favaro Simon Fraser University, Burnaby, British Columbia, Canada. reynolds@sfu.ca

\section{Dark-matter team sets record straight}

We wish to clarify a few points you make in relation to the darkmatter (DAMA) experiment at Italy's Gran Sasso National Laboratory (Nature 485, 435-438; 2012).

It is not an assumption of ours that Earth's velocity through dark matter varies as it orbits the Sun to produce an annual variation in the flux of dark-matter particles: this property was described many years ago (see, for example, K. A. Drukier et al. Phys. Rev. D 33, 3495-3508; 1986). Our experiments are based on this principle.

The XENON100 experiment, which you quote as failing to detect weakly interactive massive particles (WIMPs) in DAMA's mass range, has not been a "source of tension" for us, for both experimental and theoretical reasons. To list a few, XENON100 uses a different methodology, a different target material and several datasubtraction procedures.
The XENON100 comparisons depend on modelling, whereas our results are model-independent. Also, the sensitivities of the experiments to the many possible dark-matter candidates and to astrophysical, nuclear and particle-physics scenarios are quite different from those of DAMA, including to WIMPs scenarios.

Neither are the XENON100 results backed up by those from CRESST (G. Angloher et al. preprint at http://arxiv.org/ abs/1109.0702; 2011) at Gran Sasso, although they too search for recoil-like events in the data. Rita Bernabei ${ }^{\star}$ Tor Vergata University of Rome and National Institute of Nuclear Physics (INFN), Rome, Italy. rita.bernabei@roma2.infn.it ${ }^{*}$ On behalf of the DAMA collaboration.

\section{Evolution blackout in South Korea}

We were surprised by the creationists' victory in persuading the South Korean government to reduce the already scanty teaching of evolution in the country's schools and universities (Nature 486, 14; 2012).

Every year we interact with aspiring graduate students in ecology, evolution and behaviour who have never taken courses in these subjects. Many professionals in South Korea are unfamiliar with the theory of evolution.

In response to objections by researchers, the government decided in June to include a board of scientists in a review of their proposed changes to textbooks.

We hope that raising public awareness of this issue will help scientists and educators in South Korea to fight for the teaching of evolutionary theory in more comprehensive science curricula at schools and universities.

Sang-im Lee, Piotr Jablonski Seoul National University, Seoul, South Korea. snulbee@behecolpiotrsangim.org 